\title{
ACERCAMIENTOS Y CONTROVERSIAS SOBRE EL ESTUDIO DE LA LITERATURA FANTÁSTICA ACTUAL
}

Virginia Caamaño Morúa

\section{(c) $($ () $\odot$}

Esta obra está bajo una licencia Creative Commons

Reconocimiento-No Comercial-Sin Obra Derivada 



\title{
ACERCAMIENTOS Y CONTROVERSIAS SOBRE EL ESTUDIO DE LA LITERATURA FANTÁSTICA ${ }^{1}$ ACTUAL

\author{
APPROACHES AND CONTROVERSIES ON THE STUDY OF \\ CONTEMPORARY FANTASTIC LITERATURE
}

\section{Virginia Caamaño Morúa}

\begin{abstract}
RESUMEN
En este trabajo, se realiza un acercamiento puntual a algunas propuestas recientes para el estudio de la literatura fantástica, provenientes de críticos especializados, tanto de América como de Europa. Estos estudiosos pretenden identificar los rasgos específicos de esa literatura con el propósito de definirla, mostrando algunos de ellos la necesidad de ampliar o modificar la visión establecida por Todorov en 1970. Tales objetivos han resultado una ardua labor que aún continúa, pues los especialistas no han logrado llegar a un acuerdo, ni siquiera en cuanto a si debe considerársela un género o abordarla desde otras perspectivas.

Palabras clave: literatura fantástica, literatura latinoamericana, teoría literaria, crítica literaria,
\end{abstract} literatura actual.

\begin{abstract}
This piece specifically approaches some recent proposals for the study of fantasy literature originated by specialized critics, from both America and Europe. These scholars seek to identify the particular characteristics of this type of literature with the purpose of defining it; with some of them demonstrating the necessity of broadening or modifying the viewpoint established by Todorov in 1970. These objectives have resulted an ongoing arduous task, as specialists have not been able to agree even on whether fantasy literature should be regarded as a genre in itself, or studied from other perspectives.

Key words: fantasy literature, Latin American literature, literary theory, literary criticism, current literature.
\end{abstract}

M.L. Virginia Caamaño Morúa. Universidad de Costa Rica. Profesora Catedrática e investigadora en la Escuela de Filología, Lingüística y Literatura y en la Maestría de Literatura Latinoamericana. Costa Rica.

Correo electrónico: vicaamano@yahoo.com 


\section{[...] más allá de la realidad -la del mundo que nuestros lenguajes configuran-, el territorio, ininteligible, de lo real. \\ (González-Requena, 1998, p. 10)}

\section{Introducción}

En su acercamiento al estudio de lo fantástico, los especialistas coinciden en señalar que este surge a partir de la confrontación de dos órdenes opuestos; cuando el mundo cotidiano y normal en que se vive es perturbado por un hecho imposible y por lo tanto incomprensible, el cual rompe las normas y seguridades construidas para comprender la realidad, desplazando y sumiendo en la inestabilidad y en la inquietud tanto a los personajes como a quien lee -ya sea el lector implícito o el real- (cfr. Roas, 2011).

La contraposición entre el hecho fantástico y la realidad ha llevado a los estudiosos de estos temas por caminos diferentes. Están quienes consideran que la única realidad que importa es la instaurada intratextualmente e ignoran el contexto sociocultural y también los que han visto la necesidad de indagar sobre las relaciones que los textos establecen con la realidad empírica, en función de las diferentes maneras desde las que esta puede ser comprendida, como se verá más adelante.

Por otro lado, existe acuerdo entre un buen número de investigadores, quienes reconocen como un aporte fundamental el estudio realizado por Sigmund Freud sobre lo siniestro, a partir del cual se puede explicar el surgimiento de lo fantástico como una ruptura del orden establecido. Lo siniestro para Freud (1981, p. 2484) es "aquella suerte de espantoso que afecta las cosas conocidas y familiares desde tiempo atrás"2, y que al surgir, provoca un desequilibrio de lo cotidiano, pues muestra los límites que separan a la realidad de lo imposible, permitiendo la irrupción de lo fantástico (cfr. Roas, 2001; Bravo, 1988). Este es evanescente, inasible e inexplicable y surge ante la conciencia humana como incomprensible, confrontando a quien lee.

En parte por su carácter evanescente, la literatura fantástica fue considerada durante largo tiempo un tipo de escritura menor, excluida del canon literario, al contrario de la literatura realista, constituida a lo largo de los dos últimos siglos como la más importante y "verdadera". Sin embargo, la constancia con que lo fantástico ha sido escrito y la necesidad por parte de críticos y críticas literarias de acusar recibo de esa amplia producción, por medio de análisis y estudios, la ha visibilizado cada vez más, dejando al descubierto una gran calidad estética e imaginativa, de manera que en la actualidad se ha convertido en una de las formas literarias más leídas y estudiadas.

En América Latina, la escritura de lo fantástico no ha sido predominante, no obstante lo cual tiene una historia destacada, que empieza a mediados del siglo XIX en Argentina, con las leyendas, relatos y "Coincidencias" de Juana Manuela Gorriti (1818-1892) y las narraciones de Eduardo Wilde (1844-1913) y Eduardo Ladislao Holmberg (1852-1937), así como las de Juan Montalvo (1832-1889) en Ecuador. Sus historias se inspiran en supersticiones populares, cuyas fuentes son las antiguas culturas de cada país, las creencias cristianas, así como ciertos temas filosóficos y debates intelectuales de la época (cfr. Hahn, 1998, p. 14).

Más tarde, numerosos autores y autoras ${ }^{3}$, provenientes de diversos países e inscritos en distintos tiempos históricos y movimientos estéticos, continúan con la escritura de estos textos hasta llegar a nuestros días, cuando una larga lista de artistas continúa renovando y enriqueciendo la producción de lo fantástico en el continente, reescribiéndolo cada quien desde su propia imaginación y utilizando diversas estrategias para lograrlo. 


\section{Acercamientos}

De modo similar a lo ocurrido en relación con la escritura de lo fantástico, el estudio de esta literatura en el continente es también tempranero y al igual que en Europa, realizado mayormente por quienes la escriben, por esto en sus críticas la privilegian por encima de la literatura realista. Esto se aprecia en las páginas que le dedica el argentino Jorge Luis Borges, uno de sus más reconocidos escritores, quien en una conferencia sobre el tema, dictada en diciembre de 1949, afirma que aunque se tiene la equivocada idea de que la literatura realista está en el origen de "la verdadera literatura", lo cierto es que esta se empieza a elaborar apenas a principios del siglo XIX, mientras las literaturas de todas las culturas del mundo comienzan con relatos fantásticos.

Ya desde 1932, en un artículo titulado "El arte narrativo y la magia", Borges deja muy claramente establecida su poética sobre el arte narrativo como artificio, donde rechaza el realismo literario y prepara a los lectores para la recepción de sus ficciones, publicadas a partir de 1933, entre las que se encuentran "Las ruinas circulares" (1940), "La biblioteca de Babel" (1939), "El jardín de los senderos que se bifurcan" (1944) y "El Sur" (1953). Según explica el crítico uruguayo Rodríguez-Monegal (1976), la poética narrativa de Borges está fundamentada en su visión de que el mundo real representa un caos, ante el cual la ficción sólo tiene dos caminos: imitarlo y caer en la mímesis o establecer su propio orden, como hace la magia. Comentando el mencionado artículo Rodríguez Monegal señala que en este, Borges concluye:

\footnotetext{
[...] postulando la analogía de dos procesos causales: el de la magia,el de la narrativa. Al hacerlo, Borges explícitamente denuncia la simulación poética dela novela psicológica y en forma implícita, denuncia también la simulación de la novela realista. Una narrativa mágica queda fundamentada aquí, pero una narrativa en que el término mágico poco o nada tiene que ver con el uso que desde Franz Roh y Massimo Bontempelli, hasta Arturo Uslar Pietri, Alejo Carpentier y Miguel Ángel Asturias [...] se le viene dando en la crítica contemporánea. (Rodríguez-Monegal, 1976, pp. 182-183)
}

Ocho años después, en el prólogo a la novela La invención de Morel (1940) de su amigo Adolfo Bioy Casares, Borges confirma aún más su posición, al mantener su crítica a la novela psicológica y al realismo literario, estimándolos desordenados y afirmando que han olvidado su carácter de "artificio verbal". Sobre ellos comenta con ironía: "Los rusos y los discípulos de los rusos han demostrado hasta el hastío que nadie es imposible: suicidas por felicidad, asesinos por benevolencia, personas que se adoran hasta el punto de separarse para siempre, delatores por fervor o por humildad. Esa libertad plena acaba por equivaler al pleno desorden" (Borges, 1999, p. 29). Denuncia de esta manera a la literatura realista, a la cual juzga tan artificial y tan ficticia como la fantástica y escoge entre ambas la segunda, por su rigor, orden y lucidez.

Coincidiendo con Borges, en el prólogo de la Antología de literatura fantástica (1940) Bioy Casares considera que "las ficciones fantásticas son anteriores a las letras" y se encuentran en el origen de las literaturas de todas las culturas del mundo. Para demostrarlo, menciona como ejemplos la antiquísima novela china Sueño del aposento rojo, el Zend Avesta, La Iliada, La Biblia y las Mil y una noches y las presenta como textos habitados por lo fantástico. Pero no se queda allí, pues además incluye dentro de su visión de lo fantástico a las novelas realistas y los textos filosóficos, los cuales en su opinión, "abundan en sueños y fantasmas" (Bioy-Casares, 1997, p. 5).

Julio Cortázar (1989), por su parte, también rechaza la literatura realista, al considerar que la realidad que plasma es muy pobre. Para este autor, la realidad cotidiana es mucho más profunda y extraordinaria de lo que se cree y por medio de los intersticios que la horadan, lo insólito, lo fantástico, puede escaparse en el momento menos pensado, volviéndola inestable y 
haciendo que la supuesta normalidad anterior sea irrecuperable, tal y como sucede en sus relatos “Carta a una señorita en París" (1951), "Lejana" (1951) y "La noche boca arriba" (1956), entre muchos otros. Desde la perspectiva de Cortázar, entonces, la literatura realista es insuficiente para aprehender esa realidad que nos envuelve totalmente, sin que queramos acusar recibo de ella. Ante esto, invita al lector a ser valiente y aceptar lo fantástico sin temor, como un medio para lograr vislumbrar el mundo en que vivimos.

En virtud de las propuestas de Borges, discípulo de Macedonio Fernández, quien ya desde las décadas de los años veinte y treinta del siglo pasado había realizado novedosas propuestas de ruptura en relación con la literatura realista ${ }^{4}$; de Bioy Casares, de Cortázar y de numerosos autores más que no es posible comentar aquí, es claro que en América Latina la reflexión sobre la literatura fantástica es profunda y de larga data.

Pero no es sino hasta 1970, con la sistematización sobre su estudio realizada por el rumano Tzvetan Todorov (2006), en su Introducción a la literatura fantástica, que ella ocupa realmente un lugar central como objeto de análisis de la crítica especializada en ambos lados del Atlántico, aunque como señala la estudiosa Boccuti (2008), desde entonces hasta el presente, dadas las múltiples facetas que presentan los textos, ni siquiera ha habido coincidencia en cuanto a si lo fantástico debe considerarse un género (Todorov, 2006), una lógica narrativa (Bessière, 2001) o un modo de representación (Jackson, 2001). (cfr. Boccuti, 2008).

En el recorrido por la literatura fantástica del siglo XIX que realiza en su texto, Todorov (2006) considera que la condición indispensable para que surja lo fantástico, es la ocurrencia brusca en la realidad textual de un hecho inexplicable y como consecuencia, la vacilación del lector implícito -y a veces también del personaje- sobre la naturaleza del fenómeno ocurrido. Para este autor, fiel representante del estructuralismo de la época en que surge su estudio, lo fantástico aparece como una cualidad inmanente del texto, de manera que intenta explicarlo intratextualmente, a partir de su funcionamiento, sin confrontarlo en ningún momento con algún concepto de realidad ${ }^{5}$, que lo obligaría a acudir al contexto sociocultural desde donde sería posible observar la irrupción del fenómeno sobrenatural.

Todorov (2006) propone una clasificación tripartita a partir de los hechos narrados y la observación que de ellos hace el lector implícito. De manera que se podrían considerar hechos extraños, cuando se los puede explicar como naturales; hechos maravillosos cuando lo ocurrido es irreal o sobrenatural y no se problematiza su ocurrencia y fantásticos, en el límite entre los dos anteriores, cuando se mantiene la duda, la vacilación sobre lo ocurrido, de modo que no se pueda explicar -como en lo extraño- ni se acepte sin discusión -como en lo maravilloso-. Lo fantástico sería así de muy corta duración, se desvanecería en el instante en que se acaba la vacilación del lector -no necesariamente la del personaje- para terminar convirtiéndose en extraño o maravilloso. Para Todorov por consiguiente, esa duda del lector, así como la imposibilidad de interpretación poética o alegórica en la manera de leer lo fantástico, son condiciones indispensables para que este surja. Estas propuestas han provocado la crítica de numerosos especialistas quienes las declaran simples, vagas y restrictivas de lo fantástico. Es evidente, además que no considera en ningún momento que la autonomía del texto literario en relación con la realidad es relativa y no absoluta.

\section{Controversias}

Dos años después de la publicación del texto de Todorov, Barrenechea (1972), en su "Ensayo de una Tipología de la literatura fantástica", expresa su desacuerdo con las propuestas del rumano, afirmando que por su rigidez se elimina buena parte de la producción fantástica 
contemporánea, limitándose a la literatura producida en el siglo XIX; asimismo advierte que esa clasificación no es pertinente para el estudio de la producción literaria latinoamericana contemporánea, donde lo fantástico ha sido reapropiado y renovado por escritores y escritoras, infundiéndole características distintas de las tradicionales.

Barrenechea (1972) inicia su ensayo argumentado que la poesía ${ }^{6}$ y la alegoría pueden ser fantásticas, contrario a lo establecido por Todorov (2006). Determina luego lo fantástico como un subgénero compuesto por tres categorías, las cuales clasifica a partir de lo narrado en: hechos a-normales, a-naturales o irreales y sus contrarios y establece además la problematización o no problematización de este contraste. Para esta autora, lo maravilloso sería lo a-normal sin problematización y lo no a-normal, lo posible (cfr. Barrenechea, 1972, pp. 392-393). Barrenechea también rechaza la tesis de Todorov (2006) en cuanto a la vacilación o duda del lector como fundamento del género y en artículos posteriores reconoce además, la necesidad de valorar lo fantástico en relación con el contexto sociocultural, para conseguir determinar de modo lógico y necesario lo a-normal en el texto.

A pesar del reconocimiento que le ha significado su aporte al estudio de esta literatura, Barrenechea (1972) es a su vez criticada pues algunos de los ejemplos que presenta parecieran violentar sus propios presupuestos, según afirma Ana María Morales (cfr. 2000), mientras David Roas (2001) señala la ausencia de lo sobrenatural en una de las categorías que establece, con lo cual elimina la posibilidad de incluirlo dentro de ese tipo de literatura, donde el surgimiento de un fenómeno de esa índole es, para él, imprescindible.

Manteniendo el enfoque en la relación entre la realidad y lo fantástico, en 1974, la crítica Bessière (2001) indica que las dificultades presentadas para estudiar estos textos se asientan en la exclusión de toda referencia a su contenido semántico y en la ignorancia de su arraigo cultural. La autora niega que lo fantástico sea un género literario o una categoría y lo establece como una lógica narrativa que "refleja, bajo el aparente juego de la invención pura, las metamorfosis culturales de la razón y del imaginario colectivo" (Bessière, 2001, p. 84). En ese sentido, lo fantástico según ella nace de la mitología, la religión, la psicología y no se distingue "de las manifestaciones aberrantes de lo imaginario o de sus expresiones codificadas en la tradición popular" (Bessière, 2001, p. 84).

Como se puede apreciar, Bessière (2001) se va al otro extremo de Todorov (2006), al definir lo fantástico como "reflejo" de una realidad de la cual surge, deslegitimando su posible apreciación como productividad artística, ya que desde su propuesta, esta "lógica narrativa" aparece como muestra de una imaginación liberada y es una de las mejores expresiones de la contracultura, al convertirse en discurso colectivo que enuncia todo lo que no puede decirse en la cultura oficial. Descarta así, por otro lado, la acusación que se le ha realizado a esta práctica de ser una literatura poco "comprometida" y "escapista", según apreciación de algunos críticos.

Otro investigador que ha realizado una rica contribución al estudio de este tema es el venezolano Víctor Antonio Bravo (1988) quien expone, en primer lugar, que lo fantástico es una "de las formas de la alteridad que se encuentra en la génesis de todo acontecimiento narrativo" (Bravo, 1988, p. 7), para señalar, a continuación, que surge con la puesta en escena de dos ámbitos y la transgresión del límite que los divide (cfr. Bravo, 1988, p. 39). Bravo afirma que cuando lo fantástico persiste en el texto se vuelve insostenible y lleva a la locura, entonces se resuelve en el asombro, el cual se transforma en horror, o en la ausencia de asombro que genera el absurdo, en ambos puede manifestarse lo siniestro. Tanto el horror, que surge generalmente como expresión del mal, como el absurdo, tienen una salida en la irrisión. Lo fantástico para Bravo (1988), sólo logra mantenerse en el discurso paródico como 
una de sus marcas, a través de lo hiperbólico, lo escatológico o con el empleo de la digresión. De manera que lo paródico aparece como una nueva forma de realismo que al mismo tiempo lo niega como tal y también facilita la aparición de lo siniestro.

Al rechazar la negación de Todorov (2006) sobre la posibilidad de leer lo fantástico desde la alegoría, Bravo (1988) realiza un acercamiento a esta figura literaria y explica la metamorfosis sufrida por ella, la cual se lleva a cabo a través de la utilización de la parodia, que le sirve como medio de escape de su fijeza característica. Así indica que "[...] gran parte de la narrativa contemporánea pone en escena lo fantástico, en tanto que espesor y sustentación de una 'red alegórica' (como diría Blanchot), propuesta como una nueva forma de realismo, como una nueva indagatoria de lo real" (Bravo, 1988, p. 236). Desde estos enunciados, el autor incorpora elementos que permiten ampliar el corpus de lo fantástico, para incluir numerosos textos latinoamericanos -que se fundamentan en la alegoría- y enriquecer las posibles perspectivas desde las cuales esta escritura puede ser estudiada.

La crítica británica Rosie Jackson (2001) en su artículo "Lo 'oculto' de la cultura"7 también confronta a la literatura realista con la fantástica y expone cómo en su opinión, la crítica literaria ha acallado a la segunda con el fin de neutralizar su intento por subvertir la cultura dominante, lo cual considera un "gesto ideológico significativo, no muy distinto del silenciamiento de lo irracional por parte de la cultura" (Jackson, 2001, p. 143).

Lo fantástico, identificado con lo irracional, aparece entonces como una especie de lenguaje del inconsciente, considerado así como lo "otro" y explicado por Jackson como "lo oculto’ de la cultura, una forma de oposición social subversiva, contrapuesta a la ideología que domina en la época en que surge y que señala el camino de lo no visto y lo no dicho de esa cultura, al mostrar un hecho imposible dentro de ella, deviniendo así en subversivo.

La crítica canadiense Nandorfy $(2001)^{8}$ interpreta esta propuesta de Jackson (2001) como una intención por despojar de su poder subversivo a lo fantástico, al intentar absorber el discurso del otro en la conciencia, de modo que trascienda los elementos de ruptura y pueda servir así a la "verdadera cultura", identificada por la británica como dominante y represiva, de manera que lo fantástico en Jackson (2001) es para Nandorfy una fuerza nihilista (cfr. Nandorfy, 2001, pp. 253-255).

Al igual que la gran mayoría de estudiosos y estudiosas sobre este tema, Nandorfy (2001) también vuelve al texto de Todorov (2006) e infiere que este se adentra en un "callejón sin salida lógico para salvar la existencia de la realidad" cuando afirma "que el psicoanálisis ha invalidado nuestra necesidad de literatura fantástica" (Nandorfy, 2001, p. 252). Con esto, expone una oposición entre realidad y literatura, donde sólo es posible que exista uno de ellos, pues interpreta las experiencias modernas desde un punto de vista exclusivamente racional, impidiendo que lo fantástico sea parte y extienda nuestra noción de realidad (cfr. Nandorfy, 2001, p. 252), la cual, según esta crítica, sólo se experimenta a partir denuestra interacción con ella.

En su interpretación, Nandorfy (2001) homologa el modo en que Todorov (2006) simplifica la realidad, limitándola a lo racionalmente cognoscible, con la manera en que Jackson (2001, p. 254) "simplifica la noción de cultura al identificarla con el poder de una ideología dominante y represora", empobreciendo las múltiples vertientes de lectura que debieran darse en lo fantástico.

Nandorfy rechaza la propuesta todoroviana de que el lenguaje por sí mismo pueda crear "entidades totalmente fantásticas carentes de existencia" (Nandorfy, 2001, p. 248) que estén fuera de la realidad, pues esto implicaría que no somos parte de esa realidad. En cambio, 
propone que dada la indeterminación y polisemia que caracterizan el lenguaje, se facilita el establecimiento de lo fantástico, el cual debe verse en contraposición con la realidad, por lo cual es imprescindible definirla.

Para realizar dicha definición, plantea su propuesta fundamental desde la mecánica cuántica a partir de una revisión de las ideas del físico estadounidense David Bohm ${ }^{9}$, quien afirma que la realidad "es todo aquello que es pensable", lo cual no equivale a "lo que es", pues ninguna idea puede capturar el sentido de esto (cfr. Nandorfy, 2001, p. 244). Al no reconocerse una realidad objetiva, ya que la noción que se utiliza incluye niveles de experiencia diferentes, lo irracional y el inconsciente serían "[...] modos de ser legítimos en la infinita variedad de posibilidades que la indeterminación del lenguaje pone de manifiesto" (Nandorfy, 2001, p. 257). La autora confiesa, sin embargo, que las lenguas indoeuropeas, ligadas íntimamente con la lógica, presentan obstáculos importantes a este planteamiento. No obstante, su acercamiento pareciera brindar ricas posibilidades para el estudio del tema.

Finalmente, el escritor y ensayista español David Roas (2001) en su artículo "La amenaza de lo fantástico" establece que este debe estar forzosamente ambientado en la realidad cotidiana, apareciendo como una '[...] especie de 'hiperrealismo', puesto que, además de reproducir las técnicas de los textos realistas, obliga al lector a confrontar continuamente su experiencia de la realidad con la de los personajes: sabemos que un texto es fantástico por su relación (conflictiva) con la realidad empírica" (Roas, 2001, p. 26), a la cual transgrede. Dicha transgresión es la que marca la identidad del texto como fantástico, lo cual se manifiesta, además, en el lenguaje utilizado, el cual se vuelve oscuro, torpe, indirecto. De manera que este tipo de literatura en opinión de Roas (2001), es profundamente subversivo no sólo en el aspecto temático, sino también en el nivel lingüístico, aspecto destacado especialmente en la narrativa fantástica latinoamericana.

Para Roas (2001), como también para Bravo (2005), el miedo o al menos la inquietud, son parte esencial de los textos fantásticos actuales y llevan en última instancia a quien lea, a cuestionar la realidad en que vive. Así, la extrañeza o inquietud que surge induce a lectores y lectoras a dudar de esa convención social llamada realidad y los lleva a pensar en su posible anormalidad, de manera que se descubre que "[...] nuestro mundo no funciona tan bien como creíamos [...]” (Roas, 2001, p. 37), tal y como plantean en sus narraciones Borges, Cortázar y otros muchos escritores y escritoras.

Posteriormente, en Tras los límites de lo real, pretendiendo conciliar distintas posiciones, Roas (2011) afina y sintetiza sus propuestas, en un intento más por definir lo fantástico y lo considera una categoría estética, un discurso "en relación intertextual constante con ese otro discurso que es la realidad, entendida siempre como una construcción cultural" (Roas, 2011, p. 9), de acuerdo con lo cual,"lo fantástico se construye a partir de la convivencia conflictiva de lo real y lo imposible" (Roas, 2011, p. 45). Al evitar verlo como género -con los preceptos de forma y contenido que lo ordenarían categóricamente- y las limitaciones que esto conlleva, Roas estudia lo fantástico desde cuatro conceptos centrales que son: la realidad, lo imposible, el miedo y el lenguaje, facilitando que se lo vea desde una perspectiva multidisciplinaria que enriquece su estudio y mantiene su vigencia.

Además, de modo coherente y de acuerdo con su época, Roas (2011) reconoce la necesidad de problematizar la noción única de realidad concebida hasta inicios del siglo XX, enfrentándola con las numerosas transformaciones ocurridas en el paradigma científico a partir de una serie de descubrimientos y propuestas que se han dado en la física, en la neurobiología y aún en la filosofía, las cuales aunadas a las teorías sobre el cosmos y la influencia de las nuevas 
tecnologías de la comunicación, lo llevan a postular dos ideas fundamentales: primero, que la realidad es una construcción subjetiva y segundo, que como tal es compartida socialmente, es una construcción social. Por lo tanto, afirma Roas, "no hay realidad real", solo se conocen representaciones de ella (cfr. Roas, 2011, p. 26).

Frente a semejante relativización, lo fantástico obliga a que los hechos descritos se confronten constantemente tanto "con la lógica construida en el texto como con esa otra lógica -también construida- que es nuestra visión de lo real". Esas dos realidades paralelas, alternativas no pueden"convivir" de manera que cuando se encuentran en la normalidad aparente en que los personajes viven, análoga a la de quien lee, "se vuelve extraña, absurda e inhóspita" (Roas, 2011, p. 42), señalando la irrupción de lo fantástico.

Roas reflexiona, al final, sobre cómo la lectura de los relatos fantásticos actuales inducen a contrastar y problematizar los fenómenos imposibles que allí se narran con el "precario orden o desorden en el fingimos vivir más o menos tranquilos" (Roas, 2011, p. 177). Destaca, así, el poder de representación y el valor subversivo de esta literatura, que como dice Borges es símbolo de nosotros y de nuestra vida.

\section{3. (In) Conclusión}

Como se ha visto en este corto y parcial 'estado de la cuestión' sobre el estudio de lo fantástico en la actualidad, desde el comentado aporte de Todorov (2006) y a lo largo de más de cuarenta años, un respetable número de estudiosos han intentado definir la literatura fantástica. Sin embargo, a pesar de los trabajos serios y detallados realizados tanto en América como en Europa, no se ha logrado llegar a un acuerdo, sobre la manera más pertinente de clasificar textos tan heterogéneos en cuanto a sus rasgos y características, lo cual desde mi punto de vista no es esencial ni mucho menos negativo para el estudio de la literatura fantástica, pues la búsqueda de definiciones finales y las ansias por nominar las producciones culturales, devienen las más de las veces en prácticas fundamentalistas, limitantes y excluyentes. Inconclusivamente entonces, coincido plenamente con Nandorfy cuando afirma que:

[...] toda definición es una aproximación, que requiere la elaboración de contextos múltiples y una conciencia de que el lenguaje es de naturaleza polisémica y mutante.La etiqueta "literatura fantástica" no puede reducirse a una fórmula, puesto que designa entidades que requieren una constante definición. [...] El significado más preciso de lo "fantástico", ya sea en un contexto científico o artístico será, pues, 'potencial'; un potencial susceptible de actualización en la experiencia y en la expresión, en tanto en cuanto no se vea sometido a la práctica racional de la exclusión. (Nandorfy, 2001, p. 259)

\section{Notas}

1. En este estudio, se va a entender la literatura fantástica desde una perspectiva muy amplia, donde pueden incluirse la literatura gótica (considerada iniciadora de lo fantástico moderno, a partir del romanticismo), la ciencia ficción y otras vertientes de lo fantástico.

2. Llamado por Freud "Umheimlich", Bravo (1988, p. 36) lo asimila con lo "(espantable, angustiante, espeluznante, sospechoso, de mal agüero, lúgubre, inquietante, fantástico, demoníaco...) se encuentra caracterizado por la irrupción de algo que se encontraba reprimido y su presencia supone la presencia del peligro y de intensiones (sic) malévolas".

3. Una nómina no exhaustiva de autores y autoras de América Latina que escriben literatura fantástica se encuentra en mi artículo "Seres monstruosos y cuerpos fragmentados. Sus representaciones en dos relatos fantásticos de Alberto Chimal”. [2014]. Revista de Filología y Lingüística. 39 (1), 159-178. 
4. Cuando defiende la irrealidad del arte en su texto "Para una teoría del arte" (1927) y en obras literarias como No todo es vigilia la de los ojos abiertos (1928), Papeles de Recienvenido (1929) y sobre todo en "Novela de la Eterna", y la Niña del dolor, la "Dulce-persona" de un amor que no fue sabido (1938), anticipo de Museo de la Novela de la Eterna (1967).

5. Todorov se limita a reconocer la realidad: "tal como ella existe para la opinión común” (Todorov 2006, p. 41), de manera que aparece como ahistórica.

6. Barrenechea pone entre sus ejemplos de lo poético fantástico los poemas de Jorge Luis Borges "El Golem" y "La noche cíclica" y señala que para este autor "no hay género que no pueda alojar lo fantástico" (Barrenechea, 1972, p. 394).

7. Publicado en 1981 con el título de "Afterword: the 'Unseen' of Culture".

8. El título original de su artículo es "Fantastic Literature and the Representation of Reality", publicado en Revista Canadiense de Estudios Hispánicos. (16), 99-112.

9. David Bohm (1917-1992) hizo valiosas contribuciones al desarrollo de la mecánica cuática y la teoría de la relatividad y realizó una serie de investigaciones y descubrimientos en colaboración con otros físicos. Su diálogo con algunos filósofos permitió ampliar los alcances de sus aportes. Es considerado uno de los mejores físicos cuánticos de todos los tiempos.

\section{Bibliografía}

Barrenechea, A.M. (1972). Ensayo de una Tipología de la Literatura Fantástica. Revista Iberoamericana. 38 (80), 391- 403.

Bessière, I. (2001). El relato fantástico: forma mixta de caso y adivinanza. Por D. Roas (Ed.). Teorías de lo fantástico. (83-106). Amsterdam: Benjamins.

Bioy-Casares, A., Borges, J.L. y Ocampo, S. (1997). Prólogo. En Antología de literatura fantástica. (9-17). Buenos Aires: Editorial Sudamericana.

Bocutti, A. (2008). Humorismo y fantástico en la microficción argentina: Raúl Brasca, Rosalba Campra, Ana María Shua. Amoxcalli. (1), 223-239.

Borges, J.L. (1967). La literatura fantástica. Buenos Aires: Olivetti.

Borges, J.L. (1999). Adolfo Bioy Casares: La invención de Morel. En Prólogos con un prólogo de prólogos. (28-31). Madrid: Alianza Editorial.

Borges, J.L. (2002). El arte narrativo y la magia. En Discusión. (102-115). Madrid: Alianza Editorial.

Bravo, V.A. (1998). La irrupción y el límite. Hacia una reflexión sobre la narrativa fantástica y la naturaleza de la ficción. México: Universidad Nacional Autónoma de México.

Bravo, V.A. (2005). El miedo y la literatura. Anales de Literatura Hispanoamericana. (34), 13-17.

Cortázar, J. (1989). Historias de cronopios y famas. Argentina: Editorial Sudamericana.

Freud, S. (1981). Lo siniestro. Por J. Numhauser-Tognol (Comp.) y L. López-Ballesteros y de Torres (tr.). Obras completas. (Vol. 3, 2484-2505). Madrid: Biblioteca Nueva. (Trabajo original publicado en 1919).

González-Requena, J. (1998). En el principio fue el verbo. Palabra versus signo. Trama y Fondo. (5), 7-28. 
Hahn, O. (1998). Fundadores del cuento fantástico hispanoamericano. Antología comentada. Santiago de Chile: Editorial Andrés Bello.

Jackson, R. (2001). Lo ‘oculto’ de la cultura. Por D. Roas (Ed.). Teorías de lo fantástico. (141-153). Amsterdam: Benjamins.

Markovic, A. (2008). Lo fantástico y las normas socio-culturales. Amoxcalli. (1), 109-124.

Morales, A.M. (2000). Teoría y práctica de lo fantástico. Modelos y rupturas. Escritos. (21), 23-36.

Nandorfy, M.J. (2001). La literatura fantástica y la representación de la realidad. Por D. Roas (Ed.). Teorías de lo fantástico. (243-264). Amsterdam: Benjamins.

Roas, D. (Comp.). (2001). La amenaza de lo fantástico. En Teorías de lo fantástico. (7-44). Madrid: Arco/Libros.

Roas, D. (2011). Tras los límites de lo real. Una definición de lo fantástico. Amsterdam: Benjamins.

Rodríguez-Monegal, E. (1976). Borges: Una teoría de la literatura fantástica. Revista Iberoamericana. (95), 177-89.

Todorov, T. (2006). Introducción a la Literatura fantástica. Buenos Aires: Paidós. 